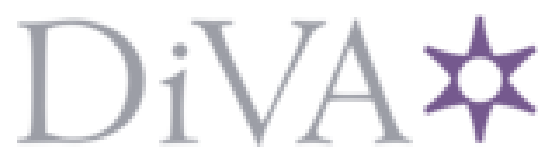

http://www.diva-portal.org

\title{
Postprint
}

This is the accepted version of a paper presented at 2014 IEEE International Symposium on Dynamic Spectrum Access Networks (IEEE DySPAN'14).

Citation for the original published paper:

Glaropoulos, I., Fodor, V. (2014)

Spectrum sharing with low power primary networks.

In:

N.B. When citing this work, cite the original published paper.

Permanent link to this version:

http://urn.kb.se/resolve?urn=urn:nbn:se:kth:diva-140530 


\section{Spectrum sharing with low power primary networks}

\author{
Ioannis Glaropoulos \\ Access Linnaeus Center \\ KTH, Royal Institute of Technology \\ Stockholm, Sweden \\ Email: ioannisg@kth.se
}

\author{
Viktoria Fodor \\ Access Linnaeus Center \\ KTH, Royal Institute of Technology \\ Stockholm, Sweden \\ Email: vfodor@kth.se
}

\begin{abstract}
Access to unused spectrum bands of low power primary networks requires a careful optimization of the secondary cooperative spectrum sensing, since the reliability of the local sensing depends significantly on the location of the cooperating nodes. In this paper we study the efficiency of cooperative sensing over multiple bands, sensed and shared by a large number of secondary users. We show that the per user cognitive capacity is maximized, if both the number of bands sensed by the secondary network as a whole, and the subsets of these bands sensed by the individual nodes are optimized. We derive the fundamental limits under different sensing duty allocation schemes. We show that with some coordination the per user cognitive capacity can be kept nearly independent from the network density.
\end{abstract}

\section{INTRODUCTION}

Cognitive radio technology, based on sensing the radio environment and adapting the transmission strategies accordingly, may allow for efficient use of radio spectrum resources. Cognitive radio networks $(\mathrm{CRN})$ can efficiently control the interference among the competing networks [1], or provide secondary access to a spectrum band, ensuring that the incumbent, primary users do not experience severe performance degradation [2].

Cognitive networks gain information on the availability of the radio spectrum through spectrum sensing or by accessing some spectrum database, decide about their spectrum access strategy based on this information, and perform interference management to control the interference caused to the other networks in the area. Therefore, the efficiency of the cognitive network depends both on the accuracy of the spectrum availability information and the efficiency of the channel access and interference management.

In this paper we consider the specific case of secondary access, and focus of the efficiency of spectrum sensing performed by the nodes of the cognitive network. Spectrum sensing has the significant advantage that the provided information is timely. Unfortunately, spectrum sensing performed locally at the SUs can not give accurate information about the spectrum availability due to the impairments of the wireless channel and the hardware limitations of the sensors [3]. Therefore, to increase sensing performance, cooperative sensing is required, where sensing results, exchanged among several sensing nodes, are combined, in order to reliably detect the presence of primary transmissions [4][5][6].

We study the efficiency of cooperative sensing when the primary transmission power is in the same order of magnitude as the secondary one. Spectrum sensing and secondary access are challenging in this scenario, since the local sensing performance degrades rapidly as the distance between the primary transmitter and the secondary sensing node increases, and at the same time a large area around the primary receiver needs to be protected, to avoid harmful secondary interference.

As the nodes of the secondary network both perform sensing and aim at utilizing the discovered spectrum bands, we evaluate the effect of the secondary user density on the per user achievable cognitive capacity. We show that as the network density increases, the performance of the cooperative sensing of a single band saturates, and the secondary network needs to optimize both the number of utilized bands and the number of bands sensed by a single user, to maximize the cognitive capacity.

The contribution of the paper is as follows:

- We provide an analytic framework to evaluate the efficiency of cooperative sensing in terms of the per user cognitive capacity under interference limitations, when the primary and secondary transmission characteristics are similar.

- We define and analyze sensing allocation mechanisms, spanning from limited to extended spectrum sensing and random to optimal sensing duty allocation.

- We study the fundamental limits of the cognitive capacity in highly dense cognitive networks and show how it is bounded by the constraints of local sensing performance.

- We demonstrate with numerical examples that sensing optimization can achieve significant gain in dense networks.

The paper is organized as follows. Related work is presented in Section II. In Section III we describe the networking scenario, the considered optimization problem and give the local sensing model. Section IV presents the analytic model of the capacity optimization of limited sensing under primary interference constraints. In Section V we introduce and evaluate the different sensing extension schemes. Section VI concludes the paper.

\section{RELATED WORK}

The optimization of secondary cognitive access, including sensing and channel access control is extensively studied in the literature. Here we consider the specific case of energy detection based cooperative spectrum sensing over multiple 
bands. The key issue in the design of the cooperative sensing solutions is the overhead introduced by the sensing itself and by the sensing control.

Considering the control of the cooperative sensing and the fusion of the sensing results, proposed solutions are based on a common control channel, e.g. [7], or distributed consensus protocols [8][9], demonstrating that the control overhead is not significant, due to the localized nature of the decision processes. Similarly, distributed solutions are proposed to coordinate the access to the cognitive channels [10][11].

The overhead of sensing depends on several parameters, as the frequency sensing needs to be performed with, the time needed to sense an individual band, the number of bands needed to be sensed by a single user, the granularity of the sensing results to be shared, and the efficiency of the decision combining. The frequency of the spectrum sensing affects the energy consumption overhead and, together with the time spent for sensing, gives the ratio of time that is surely lost for the secondary communication. Therefore, [12][13] optimize the sensing interval based on the primary channel access statistics. Once the sensing interval is set, the aim is to optimize the time spent for the sensing process, which typically means to find the number of bands to be sensed and the per band sensing time, such that primary interference constraints are met and the secondary sensing performance or the secondary throughput is maximized [14][15].

Sensing decision combining under cooperative sensing falls in one of two categories, hard decision combining or soft decision combining. Under hard decision combining the local decisions about the band availability are combined, with AND, OR or some k-out-of-N fusion rule, while under soft decision combining the quantized energy measurements are shared for the cooperative decision. Hard decision combining is often considered for its limited transmission overhead. As [14] and [16] show, the OR rule is typically more efficient than the AND one, while optimized decision combining outperforms these two, especially if even the local decision thresholds are carefully tuned. However, considering the effect of the average primary SNR and the number of nodes participating in the cooperative decision, all these schemes have similar behavior. Hard and soft decision combining are compared in [17], which concludes that under transmission errors the gain of soft decision combining is limited.

The allocation of sensing duties to a limited set of sensors is addressed in [18], considering a priori knowledge of the band occupancy probability and of the average SNR. The set of sensing nodes is optimized based on the experienced signal propagation environment in [19]; the proposed solution is further improved in [20], where learning is applied to select the sensing nodes taking even the sensing delay and the control traffic into account. [21] optimizes the number of users sensing a single band in a multichannel environment, recognizing that with increased fading more and more nodes need to sense the same band, which decreases the number of bands accessible for the secondary network.

Most of the studies on sensing and interference manage-

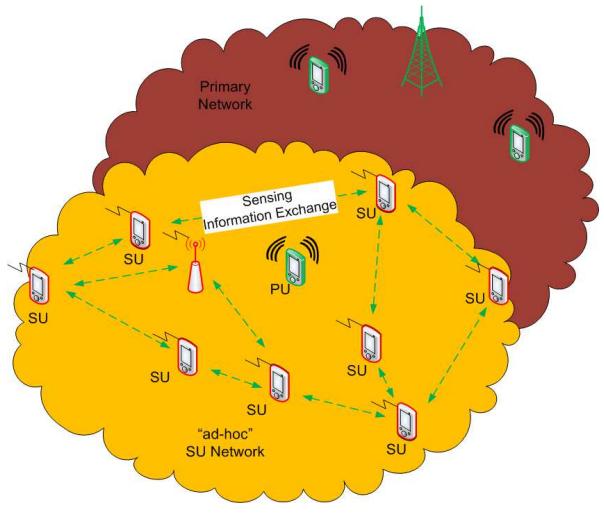

Fig. 1. Secondary Cognitive Radio Network, coexisting with the Primary Network in the same area.

ment, however, consider primary users with large transmission power, resulting in equal average SNRs at the cognitive sensing nodes. Few works are available for the scenario considered in this paper, where the cooperating SUs see significantly different average SNR levels. In [22] the authors propose a framework for cooperative sensing where local sensing is distance-dependent. Taking into account the spatial distribution of the cognitive users, the authors model the accumulative interference to a PU, without, however, considering a capacity maximization problem. In [23] a specific case of this scenario is considered, where the primary transmission range is significantly lower than the secondary one. Sensing and channel access is considered jointly in [24], however only for the scenario where the local sensing parameters are not distance dependent and the subsets of channels sensed by the given secondary users are not optimized.

\section{SYSTEM DESCRIPTION}

\section{A. Sensing and interference management}

We consider a primary and a cognitive secondary network in the same geographical area, as shown in Figure 1. The primary and the secondary transmitters have similar characteristics, thus similar transmission range. Secondary users (SUs) are randomly dispersed in the area, with density $\rho$. SUs perform spectrum sensing over a set of frequency bands with the help of their embedded sensing equipment. SUs exchange information to perform cooperative sensing, derive their relative locations [25][26], control the individual sensing processes, and coordinate the access to the cognitive bands [7]-[11].

We consider four different cases of sensing duty allocation: Under limited spectrum sensing each of the SUs senses the same set $\mathcal{M}$ of narrow frequency bands, $|\mathcal{M}|=M$. We denote $M$ as the local sensing budget. $M$ is limited by the nodes' hardware constrains, that is, $M<M_{\max }$. The goal of the sensing duty allocation optimization is to find the optimal $M$ value and cooperative sensing parameters for a given $\mathrm{SU}$ density.

Under extended spectrum sensing the SUs together aim at sensing a set of $\mathcal{W}$ bands, of size $|\mathcal{W}|=W$, denoted as the 
nominal sensing budget. Each secondary user $i$ may sense a different subset of bands, $\mathcal{M}_{i} \subset \mathcal{W}$. As nodes have similar sensing capabilities, we still consider $\left|\mathcal{M}_{i}\right|=M<M_{\max }$, $\forall i$. If the users sense the same subset, $\mathcal{M}_{i}=\mathcal{M}=\mathcal{W}$, the scenario reduces to the limited spectrum sensing. We consider three different policies for sensing duty allocation under extended spectrum sensing:

1) Random sensing: each of the secondary users selects the subset $\mathcal{M}_{i}$ of frequency bands, by picking each band of $\mathcal{W}$ with the same probability. $W, M$ and the cooperative sensing parameters are optimized for the given SU density.

2) Coordinated sensing: the secondary users coordinate the sensing duty allocation, such that each band of $\mathcal{W}$ is sensed by approximately the same number of users. Again, optimization is performed for the average user density.

3) Optimal sensing: the secondary network is aware of the instantaneous number of SUs in the area and performs dynamic sensing budget adjustment accordingly. Then, it performs coordinated sensing, considering the actual sensing budget given by the optimized $W$ and $M$.

SUs operate in a time-slotted, slot-synchronized manner. They conduct spectrum measurements and share spectrum availability information at the beginning of a time-slot, and transmit in the second part of the time-slot, if free bands have been detected. We consider hard decision combining with local energy detection, where each sensor shares only its binary only noise or signal present decision, and OR decision rule, that is, a band is considered as occupied if at least one sensor decides for signal present.

Figure 2 illustrates the main principles of the considered sensing and interference management framework. An arbitrary primary transmitter is surrounded by a prohibited area, inside which simultaneous secondary transmissions within the same frequency band would cause interference. The radius of the prohibited area, $R_{I}$, is determined by the transmission characteristics of primary and secondary users, associated with the transmission ranges $R_{p}$ and $R_{s}$ respectively. Considering the worst case scenario, when the primary receiver of the particular transmitter lies in the border of the primary transmission range, $R_{I}$ becomes $R_{I}=R_{p}+R_{s}$, as shown in the Figure. In the rest of the analysis we will assume that transmission ranges are fixed and so the radius $R_{I}$ is fixed and known to the secondary users.

To detect a transmitting PU at a given location, a subset of the SUs inside the related prohibited area performs cooperative sensing. Since the reliability of the local sensing decreases with the distance to the transmitter, we define the sensing area as a disk centered at the considered PU location. The size of the sensing area, that is, the extent of the cooperative sensing, is controlled by the cooperation radius $R_{c} \leq R_{\mathcal{I}}$. As shown in Figure 2 spectrum sensing for the considered PU location is conducted by $N_{\mathcal{A}}$ SUs inside the area $\mathcal{A}$. Area $\mathcal{B}$ is the area where existing SUs do not sense for the primary user but can

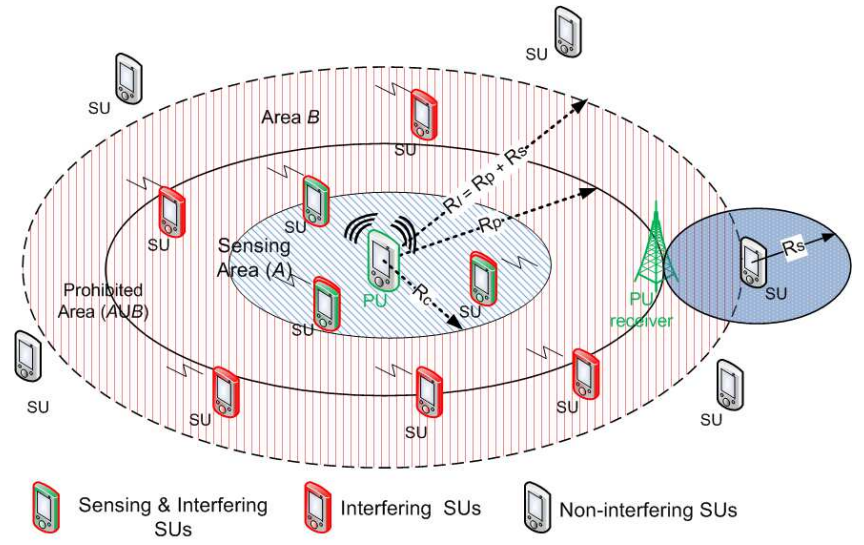

Fig. 2. Interference modelling scheme: radius $R_{I}$ defines the disk that corresponds to the prohibited area of a PU, which is determined by the communication ranges of the primary and secondary users.

cause harmful interference. The union $\mathcal{A} \cup \mathcal{B}$ constitutes the prohibited area for the considered primary user location, with $N_{\mathcal{A}}+N_{\mathcal{B}}$ SUs.

As for the cognitive transmission, we consider an idealized channel access scheme in the secondary network, where, after each sensing period, the available bands, i.e. the ones, for which cooperative sensing resulted in a correct detection of a free band or a missed detection of an occupied one, are assigned fairly to the SUs within $\mathcal{A} \cup \mathcal{B}$, with at most one band for an SU at a time.

\section{B. Sensing optimization}

Our aim is to maximize the per user cognitive network capacity, that is, the amount of spectrum resources available for each of the secondary users. The secondary users need to satisfy their bandwidth requirements through their own cooperative spectrum sensing. Their ability to extend sensing reliably to a large portion of radio spectrum enhances, as their population in a certain area increases, but at the same time, the available resources need to be shared among a higher number of SUs.

To capture this trade-off we define the per user effective cognitive capacity, $C$, as the ratio of the spectrum resources that are available for cognitive communication and the sum of resources requested by the secondary users, assuming that even a mis-detected band is useful resource for the cognitive communication. We limit $C \leq 1$, as an SU can transmit on one band only.

The secondary channel access is limited by the probability of interference at the primary user from the secondary network. To reflect the fact that channels detected free may remain unutilized if all SUs are satisfied, we define the probability of interference by the joint probability that the primary transmission is not detected and the band is assigned to a secondary user in the interference area.

We aim at optimizing the sensing duty allocation such that $\mathrm{C}$, the expected per user effective cognitive capacity under 
given SU density $\rho$ is maximized, while respecting $P_{\mathcal{I}}^{(\max )}$, the primary system interference constraint. Formally:

$$
\begin{gathered}
\operatorname{maximize} \mathbf{C} \\
\text { subject to } P(\mathcal{I}) \leq P_{\mathcal{I}}^{(\max )} \\
M \leq M_{\max },
\end{gathered}
$$

In addition, under optimal extended sensing the $N_{\mathcal{A}}$ and $N_{\mathcal{B}}$ values are known, and the optimization can be reformulated as:

$$
\begin{array}{ll}
\text { maximize } & C\left(N_{\mathcal{A}}, N_{\mathcal{B}}\right) \\
\text { subject to } & P\left(\mathcal{I} \mid N_{\mathcal{A}}, N_{\mathcal{B}}\right) \leq P_{\mathcal{I}}^{(\max )}, \\
& 0<M \leq M_{\max },
\end{array}
$$

and $\mathbf{C}$ is the average of the achieved $C\left(N_{\mathcal{A}}, N_{\mathcal{B}}\right)$ over the distributions of $N_{\mathcal{A}}, N_{\mathcal{B}}$.

There are numerous input parameters of this optimization problem and numerous system variables to be optimized. Specifically, as input parameter, we consider $R_{\mathcal{I}}$, the radius of the prohibited area; $P_{\mathcal{I}}^{(\max )}$, the primary interference constraint; the sensing parameters as $M_{\max }$, the maximum number of sensed bands, and $T_{s}$, the total sensing time; and finally, $\rho$, the secondary network density. The optimized system variables are $\gamma$, the threshold value of the local sensing; $R_{c}$, the radius of the sensing area; $W$, the number of sensed bands; and $M$, the number of bands sensed by a single SU.

Note, that we do not consider the optimization of the sensing time $T_{s}$, the length of the cognitive time slot, the fusion rule, and the overhead of cooperation, to keep the problem tractable. As discussed in Section II, related results can be found e.g., in [12]-[17].

\section{Local sensing framework}

During the sensing period an arbitrary secondary user measures the energy that is received within each of the frequency bands in its local sensing budget. Then it makes a binary decision, regarding the existence of an active primary transmission, by comparing the measured signal energy with a predefined energy decision threshold. We define the two complementary hypotheses as follows:

$$
\left\{\begin{array}{c}
\mathcal{H}_{0}: x[k]=v[k] \text { only noise } \\
\mathcal{H}_{1}: x[k]=h \cdot s[k]+v[k] \text { signal present }
\end{array}\right.
$$

where $x$ is the received primary signal, $h$ is the channel coefficient of the link between the SU and the hypothetical primary transmitter and $s$ is the transmitted signal, which is assumed to be constant for the sake of simplicity. The local test variable $\mathbf{y}$ is formed by squaring and integrating $K$ samples of the received signal during the per band sensing time, $t_{s}$ :

$$
\mathbf{y}=\frac{1}{K} \sum_{k=1}^{K}|x[k]|^{2} \underset{\mathcal{H}_{0}}{\stackrel{\mathcal{H}_{1}}{\gtrless}} \gamma_{i},
$$

where $\gamma$ is the selected decision threshold. For Rayleigh flat-fading channel with lognormal shadowing, the channel coefficient obtains the following form:

$$
h=\frac{1}{\left(r / r_{0}\right)^{\eta / 2}} e^{j \phi} \alpha 10^{\zeta / 20} .
$$

In the above expression $r$ is the distance between the hypothetic primary transmitter location and the sensing device, $r_{0}$ is a close-in reference, $\eta$ is the path-loss exponent, $\alpha, \zeta$ are a unit - variance Rayleigh and a zero-mean Gaussian random variable respectively representing small scale fading and shadowing and $\phi$ is a random phase shift uniformly distributed in $\left[-\frac{\pi}{2}, \frac{\pi}{2}\right]$. Based on (4) and (5) the probability density function of the test variable $\mathbf{y}$ under the two hypotheses is given in the following formulas:

$$
\begin{gathered}
p_{\mathbf{y}}\left(y ; \mathcal{H}_{0}\right)=\frac{1}{(\sigma / K)^{2 K} \Gamma(K)} y^{K-1} e^{y /(\sigma / K)^{2}}, \\
p_{\mathbf{y}}\left(y ; \mathcal{H}_{1} / \alpha, \zeta\right)= \\
=\frac{1}{(\sigma / K)^{2}}\left(\frac{y}{q^{2}}\right)^{\frac{(K-1)}{2}} e^{-\frac{\left(q^{2}+y\right)}{(\sigma / K)^{2}}} I_{K-1}\left(\sqrt{y} \frac{2 q}{(\sigma / K)^{2}}\right),
\end{gathered}
$$

where $q^{2} \triangleq P_{W} K \frac{10^{\zeta / 10} \alpha^{2}}{\left(r / r_{0}\right)^{\eta}} . P_{W}$ is the transmitted signal power in the considered band, i.e. $P_{W}=|s|^{2}, \sigma^{2}$ is the noise power and $I_{K}(\cdot)$ is the $K$-th order Bessel modified function of the first kind.

Local missed detection and false alarm probabilities with respect to the energy threshold $\gamma$ are given as in [24] - by averaging over the random variables $\alpha$ and $\zeta$ :

$$
\begin{gathered}
p_{m d}(r, \gamma)=\int_{0}^{\gamma} \int_{0}^{\infty} \int_{-\infty}^{\infty} p_{\mathbf{y}}\left(y ; \mathcal{H}_{1} / \alpha, \zeta\right) p_{\zeta} p_{\alpha} d \zeta d \alpha d y \\
p_{f a}(r, \gamma)=\int_{\gamma}^{+\infty} p_{\mathbf{y}}\left(y ; \mathcal{H}_{0}\right) d y
\end{gathered}
$$

Since the number of signal samples integrated at the energy detector can be consider large enough, we approximate the above density functions with Gaussian, obtaining the following simplified expressions:

$$
\begin{gathered}
\hat{p}_{m d}(r, \gamma)=\operatorname{Pr}\left(\text { no detection } \mid \mathcal{H}_{1}\right)= \\
=1-\mathbf{E}_{\alpha^{2}}\left[\mathbf{E}_{\zeta}\left[Q\left(\frac{\gamma-\sigma^{2}-\alpha^{2} 10^{\zeta / 10} P_{W}\left(r / r_{0}\right)^{-\eta}}{\sqrt{2 \sigma^{4} / K}}\right)\right]\right] \\
\hat{p}_{f a}(r, \gamma)=\operatorname{Pr}\left(\text { false detection } \mid \mathcal{H}_{0}\right)= \\
=Q\left(\frac{\gamma-\sigma^{2}}{\sqrt{2 \sigma^{4} / K}}\right), \forall i .
\end{gathered}
$$

As the received signal level depends significantly on $r$, we define the detection threshold $\gamma$ as the linear combination of the received noise and the expected signal power at the SU, given distance $r$ to the hypothetic transmitter: $\gamma: \mathbb{R}_{+} \rightarrow$ $\left(\sigma^{2}, \infty\right)$, that is:

$$
\gamma=\gamma(r)=\gamma_{0} \cdot \sigma^{2}+\gamma_{1} \cdot P_{W} r^{-\eta}
$$


Consequently, secondary users relatively close to the considered primary transmitter location apply higher decision thresholds and decrease their local false alarm probability.

To calculate the expected local false alarm and missed detection probabilities, consider the situation in Figure 2. Sensing collaboration is extended to a circular sensing area around the tested PU location, determined by the radius $R_{c}$. Since SUs are uniformly and independently distributed, the expected local missed detection probability of any SU within the sensing area is given by:

$$
\begin{aligned}
& \mathbf{p}_{m d}\left(R_{c}, \gamma\right)=\int_{0}^{R_{c}} \hat{p}_{m d}(r, \gamma) \mathbf{p}\left(r \mid R_{c}\right) d r= \\
& \quad=\int_{0}^{R_{c}} \frac{2}{R_{c}^{2}} r \hat{p}_{m d}(r, \gamma) d r,
\end{aligned}
$$

where $\mathbf{p}\left(r \mid R_{c}\right)$ denotes the probability that a secondary user lies inside the infinitely thin ring at distance $r$ from the PU (index $i$ is omitted for the sake of simplicity):

$\mathbf{p}\left(r \mid R_{c}\right)=\operatorname{Pr}\{$ user lies in the ring at distance $r\}=\frac{2 r d r}{R_{c}^{2}}$.

Similarly, secondary users cooperating within a sensing area with radius $R_{c}$ where no primary transmitters are active generate false alarm with expected local false alarm probability:

$$
\begin{aligned}
& \mathbf{p}_{f a}\left(R_{c}, \gamma\right)=\int_{0}^{R_{c}} \hat{p}_{f a}(r, \gamma) \mathbf{p}\left(r \mid R_{c}\right) d r= \\
& \quad=\int_{0}^{R_{c}} \frac{2}{R_{c}^{2}} r \hat{p}_{f a}(r, \gamma) d r .
\end{aligned}
$$

\section{MAXimizing the Cognitive CAPACITY UNDER LiMITED SENSING}

First we evaluate the efficiency of limited sensing, that is, when all SUs in the sensing area of a PU location sense the same set of narrow frequency bands. For the analysis we assume that there is only one active PU, and derive how large part of the remaining free capacity can be used by the SUs in the interference area, such that the interference limit towards the PU is respected.

\section{A. Analytic model for interference and cognitive capacity with primary user operating in a single band}

Consider that the primary user in Figure 2 transmits in band $V \in \mathcal{M}$, while all the other bands of $\mathcal{M}$ are not utilized by the primary network. The PU encounters interference, $\mathcal{I}$, on band $V$, if both $i$ ) spectrum sensing on band $V$ results in a missed detection, and ii) during the following time interval this band is assigned - based on the access scheme - to a secondary user that lies inside its prohibited area.

SUs in area $\mathcal{A}$ use the total sensing time $T_{s}$ to sense sequentially $M \leq M_{\max }$ bands. The available sensing time for any band is thus $t_{s}=T_{s} / M$.

To model the random location of secondary users, we consider the SUs' population in areas $\mathcal{A}$ and $\mathcal{B}$ be independent Poisson variables with the same expected density, $\rho$.

Following the definition in Section III-B, we express $P\left(\mathcal{I} \mid N_{\mathcal{A}}, N_{\mathcal{B}}\right)$, the probability of interference to the primary user, conditioned on the number of the existing secondary users in areas $\mathcal{A}$ and $\mathcal{B}$, as:

$$
\begin{aligned}
& P\left(\mathcal{I} \mid N_{\mathcal{A}}, N_{\mathcal{B}}\right)= \\
& \quad=\operatorname{Pr}\left\{\text { miss. det. } \mid N_{\mathcal{A}}\right\} \cdot \operatorname{Pr}\left\{\text { use } \mid N_{\mathcal{A}}, N_{\mathcal{B}}\right\} .
\end{aligned}
$$

In the following we derive the expressions for both factors in (13). Based on the $O R$ decision rule, we obtain the missed detection probability of the cooperative sensing:

$$
\begin{aligned}
& \operatorname{Pr}\left\{\text { miss. det. } \mid N_{\mathcal{A}}\right\} \triangleq p_{M D}\left(N_{\mathcal{A}}\right)= \\
& \quad=\prod_{i=1}^{N_{\mathcal{A}}} \operatorname{Pr}\{\text { miss. det. node } i\}=\left(\mathbf{p}_{m d}\left(R_{c}, \gamma\right)\right)^{N_{\mathcal{A}}} .
\end{aligned}
$$

Notice that (14) assumes uncorrelated local measurements. The assumption has been justified in [27]. Furthermore, the probability that the band $V$ will be assigned for cognitive operation to a secondary user in the prohibited area is given by:

$$
\operatorname{Pr}\left\{\text { use } \mid N_{\mathcal{A}}, N_{\mathcal{B}}\right\}=\sum_{j=0}^{M-1} \min \left\{1, \frac{N_{\mathcal{A}}+N_{\mathcal{B}}}{j+1}\right\} \cdot P_{f r}(j),
$$

where $P_{f r}(j)$ defines the probability that $j$ out of the unused $M-1$ bands are available for cognitive operation after sensing. A band may not be available for cognitive use if spectrum sensing in this band resulted in a false alarm. Since the false alarm probability is independent for each sensed band,

$$
\begin{aligned}
& P_{f r}(j) \triangleq \operatorname{Pr}\{j \text { bands detected free }\} \\
& \quad=\left(\begin{array}{c}
M-1 \\
j
\end{array}\right)\left(p_{F A}\left(N_{\mathcal{A}}\right)\right)^{M-1-j}\left(1-p_{F A}\left(N_{\mathcal{A}}\right)\right)^{j},
\end{aligned}
$$

where $p_{F A}\left(N_{\mathcal{A}}\right)$ is the false alarm probability of cooperative sensing:

$$
\begin{aligned}
& p_{F A}\left(N_{\mathcal{A}}\right) \triangleq \operatorname{Pr}\left\{\text { false alarm in a single band } \mid N_{\mathcal{A}}\right\} \\
& \quad=1-\left(1-\mathbf{p}_{f a}\left(R_{c}, \gamma\right)\right)^{N_{\mathcal{A}}} .
\end{aligned}
$$

Notice in (14) and (17) that $\mathbf{p}_{m d}\left(R_{c}, \gamma\right)$ and $\mathbf{p}_{f a}\left(R_{c}, \gamma\right)-$ given in (11) and (12) respectively - also depend on the available sensing time for each band, which is inversely proportional to the number of sensed bands, $M$. Finally, the expected interference at the primary user from the secondary network is given by the following expression:

$$
\begin{aligned}
& P(\mathcal{I})= \\
& =\sum_{N_{\mathcal{A}}=0}^{\infty} \sum_{N_{\mathcal{B}}=0}^{\infty} P\left(\mathcal{I} \mid N_{\mathcal{A}}, N_{\mathcal{B}}\right) \cdot \mathbf{p}_{N_{\mathcal{A}}} \mathbf{p}_{N_{\mathcal{B}}} \\
& =\sum_{N_{\mathcal{A}}=0}^{\infty} \sum_{N_{\mathcal{B}}=0}^{\infty}\left[P\left(\mathcal{I} \mid N_{\mathcal{A}}, N_{\mathcal{B}}\right) \cdot\right. \\
& \quad \quad \frac{(|\mathcal{A}| \rho)^{N_{\mathcal{A}}}}{N_{\mathcal{A}} !} e^{\left.-(|\mathcal{A}| \rho) \frac{(|\mathcal{B}| \rho)^{N} \mathcal{A}}{N_{\mathcal{B}} !} e^{-(|\mathcal{B}| \rho)}\right],}
\end{aligned}
$$

where $P\left(\mathcal{I} \mid N_{\mathcal{A}}, N_{\mathcal{B}}\right)$ is given by (13) based on the derivations in (14), (15) and (16) and $\mathbf{p}_{N_{\mathcal{A}}}$ and $\mathbf{p}_{N_{\mathcal{B}}}$ define the probabilities of having $N_{\mathcal{A}}$ and $N_{\mathcal{B}}$ in areas $\mathcal{A}$ and $\mathcal{B}$ respectively. These probabilities depend on the sizes of the areas, denoted as $|\mathcal{A}|$ and $|\mathcal{B}|$, corresponding to radii $R_{c}$ and $R_{\mathcal{I}}$ respectively. Note, that they do not depend on the components of $R_{\mathcal{I}}$, that is, $R_{P}$ and $R_{S}$.

The effective cognitive capacity, as defined in Section III-B, is a function of the number of SUs in areas $\mathcal{A}$ and $\mathcal{B}$, and 
depends on the number of bands detected free in $\mathcal{M} \backslash V$, and on the probability that the primary transmission on band $V$ is not detected:

$$
\begin{aligned}
& C\left(N_{\mathcal{A}}, N_{\mathcal{B}}\right) \triangleq \\
& \quad \sum_{j=0}^{M-1}\left[\min \left\{1, \frac{j}{N_{\mathcal{A}}+N_{\mathcal{B}}}\right\}\left(1-p_{M D}\left(N_{\mathcal{A}}\right)\right)+\right. \\
& \left.\quad+\min \left\{1, \frac{j+1}{N_{\mathcal{A}}+N_{\mathcal{B}}}\right\} p_{M D}\left(N_{\mathcal{A}}\right)\right] \cdot P_{f r}(j) .
\end{aligned}
$$

The expected effective cognitive capacity depends on the SU distribution, that is:

$$
\mathbf{C}=\sum_{N_{\mathcal{A}}=0}^{\infty} \sum_{N_{\mathcal{B}}=0}^{\infty} C\left(N_{\mathcal{A}}, N_{\mathcal{B}}\right) \cdot \mathbf{p}_{N_{\mathcal{A}}} \mathbf{p}_{N_{\mathcal{B}}} .
$$

Although it is not shown in (19), (20), $\mathbf{C}$ is a function of the decision threshold $\gamma$ and the cooperation radius $R_{c}$, so it is related to all of the system design parameters that we wish to optimize.

Given (18) and (19), the solution of the optimization problem (1) is not straightforward, since the objective and constraint functions are not convex, and therefore extensive search over the system variables $\gamma, R_{c}$ and $M$, is required. The search process is impeded by the fact that the feasible set of (1) is generally not compact, since $P(\mathcal{I})$ is not even monotonic with respect to the system variables. It is though possible to reduce the searching borders of $R_{c}$, within which extensive search is, however, still necessary.

First we solve a modified version of (1), with the interference constraint function, $P(\mathcal{I})$, reduced to the missed detection probability,

$$
\tilde{P}(\mathcal{I})=\sum_{N_{\mathcal{A}}=0}^{\infty} p_{M D}\left(N_{\mathcal{A}}\right)=e^{-\left(1-\mathbf{p}_{m d}\left(R_{c}, \boldsymbol{\gamma}\right)\right)|\mathcal{A}| \rho} .
$$

Now $\tilde{P}(\mathcal{I})$ is increasing with $\gamma$ and $M$, while it is decreasing with $R_{c}$. Since the cognitive capacity, $\mathbf{C}$ is decreasing with $R_{c}$ as well, the optimal radius, $\tilde{R}_{c}^{*}$, for the modified problem is given by:

$$
\tilde{R}_{c}^{*}(M, \gamma)=\tilde{P}(\mathcal{I})^{-1}\left(P_{\mathcal{I}}^{(\max )}\right), \forall M, \gamma,
$$

where the inverse function is defined with respect to $R_{c}$. Since $R_{c} \leq R_{\mathcal{I}}, \gamma$ is restricted by the set of maximal values $\gamma^{(\max )}$, which are the solutions of:

$$
\gamma^{(\max )}=P(\mathcal{I})^{-1}\left(P_{\mathcal{I}}^{(\max )}, R_{\mathcal{I}}\right),
$$

where the inverse function is taken with respect to $\gamma$.

Since $\operatorname{Pr}\left\{\right.$ use $\left.\mid N_{\mathcal{A}}, N_{\mathcal{B}}\right\} \leq 1$ and for low values of $N_{\mathcal{A}}, N_{\mathcal{B}}$ it holds $\operatorname{Pr}\left\{\right.$ use $\left.\mid N_{\mathcal{A}}, N_{\mathcal{B}}\right\}<1$ we have:

$$
\left.P(\mathcal{I})\right|_{R_{c}=\tilde{R}_{c}^{*}(M, \gamma)}<P_{\mathcal{I}}^{(\max )} .
$$

A a result, the optimal radius, $R_{c}^{*}(M, \gamma)$ for any $M, \gamma$ in (1) is lower than $\tilde{R}_{c}^{*}(M, \gamma)$.

Similarly, a lower bound for $R_{c}^{*}(M, \gamma)$ can be derived by considering a lower bound for $\operatorname{Pr}\{$ use $\}$, assuming zero false alarm probability:

$$
\hat{\operatorname{Pr}}\{\text { use }\}=\min \left\{1, \frac{N_{A}+N_{B}}{M}\right\} .
$$

This leads to:

$$
\hat{P}(\mathcal{I})=\sum_{N_{\mathcal{A}}} \sum_{N_{\mathcal{B}}} p_{M D}\left(N_{\mathcal{A}}\right) \min \left\{1, \frac{N_{A}+N_{B}}{M}\right\} \mathbf{p}_{N_{\mathcal{A}}} \mathbf{p}_{N_{\mathcal{B}}}
$$

and

$$
\hat{R}_{c}^{*}(M, \gamma)=\hat{P}(\mathcal{I})^{-1}\left(P_{\mathcal{I}}^{(\max )}\right), \forall M, \gamma .
$$

Finally, the optimal $R_{c}^{*}(M, \gamma)$ is the minimum solution of $\left.P(\mathcal{I})\right|_{M, \gamma}=P_{\mathcal{I}}^{(\max )}$ found by extensive search in a discretized version of the interval $\left(\hat{R}_{c}^{*}, \tilde{R}_{c}^{*}\right)$.

Since $\operatorname{Pr}\left\{N_{\mathcal{A}}+N_{\mathcal{B}} \leq M\right\}$ decreases with $\rho, \hat{P}(\mathcal{I})$ approaches $\tilde{P}(\mathcal{I})$, as $\rho$ increases. As a result, $\hat{R}_{c}^{*}$ approaches $\tilde{R}_{c}^{*}$, which significantly reduces the size of the search interval for the original problem in (1) for the considered dense networks.

B. Cognitive capacity and interference modelling with primary users operating in multiple bands

Let us now consider the scenario when the PU in Figure 2 utilizes a set of frequency bands. We allow this set to be random in each time slot, assuming a simple ON-OFF model for primary user activity, parameterized by the per band PU load, $w$ :

$$
\operatorname{Pr}\left\{\text { band } V_{i} \text { is occupied }\right\}=w \in(0,1), \forall V_{i} \in \mathcal{M} .
$$

Following the derivation of $P(\mathcal{I})$ in Section IV-A, only (16) has to be reformulated, since it gives $P_{f r}(j)$, the probability that $j$ bands are detected free, which now additionally depends on the number of bands occupied by the PU.

According to the activity model of (25), $\boldsymbol{v}(k)$, the probability that $k$ bands are occupied in addition to band $V$ is:

$$
\boldsymbol{v}(k)=\left(\begin{array}{c}
M-1 \\
k
\end{array}\right) w^{k}(1-w)^{M-1-k}, 0 \leq k \leq M-1,
$$

and the probability that $j$ bands are detected free is given as:

$$
P_{f r}(j)=\sum_{k=0}^{M-1} P_{f r}(j \mid k) \boldsymbol{v}(k) .
$$

Let us denote by $n$ the number of frequency bands that have been detected free, while occupied by the PU, that is detection has failed, and by $m$ the number of bands that have been successfully detected free by the SUs. The random variable $j=n+m$ gives the total number of the available bands. As the bands experience independent load, we get:

$$
\begin{aligned}
& P_{f r}(j \mid k)=\operatorname{Pr}\{j \text { bands detected free } \mid k \text { are used }\}= \\
& =\quad f_{n \mid k} * f_{m \mid k}, 0 \leq n \leq M-1, n+m=j,
\end{aligned}
$$

where $*$ denotes convolution, and $f(n \mid k)$ and $f(m \mid k)$ represent the distributions of variables $n$ and $m$ respectively $(n \leq k$, $m \leq M-1-k)$ :

$$
\begin{aligned}
f_{n \mid k} & =\left(\begin{array}{l}
k \\
n
\end{array}\right)\left(p_{M D}\left(N_{\mathcal{A}}\right)\right)^{n}\left(1-p_{M D}\left(N_{\mathcal{A}}\right)\right)^{k-n} \text { and } \\
f_{m \mid k} & =\left(\begin{array}{c}
M-k-1 \\
m
\end{array}\right)\left(p_{F A}\left(N_{\mathcal{A}}\right)\right)^{M-k-1-m}\left(1-p_{F A}\left(N_{\mathcal{A}}\right)\right)^{m},
\end{aligned}
$$

with $p_{M D}\left(N_{\mathcal{A}}\right)$ and $p_{F A}\left(N_{\mathcal{A}}\right)$ given in (14) and in (17).

The effective cognitive capacity $\mathbf{C}$ is then given by (19), with $P_{f r}(j)$ defined in (27). 


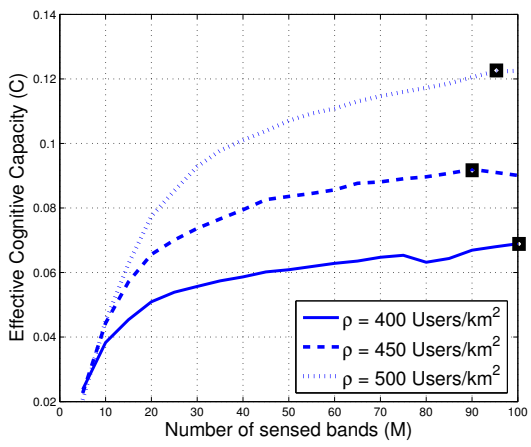

Fig. 3. Effective cognitive capacity with respect to the total number of sensed frequency bands, $M$, for various cognitive network densities. Interference limit: $P_{\mathcal{I}}^{(\max )}=10^{-3}$. Optimal values are marked.

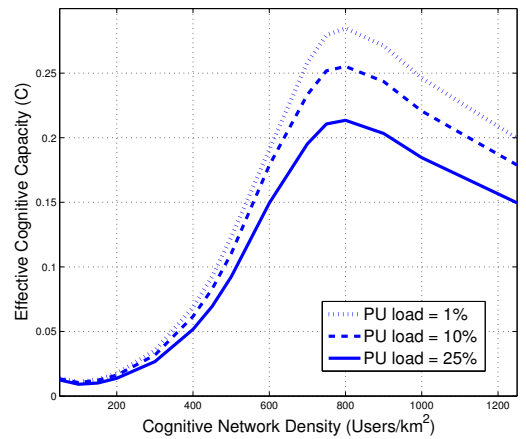

Fig. 5. Effective cognitive capacity with respect to average secondary network density, for different primary user activity levels, $w$. Interference limit: $P_{\mathcal{I}}^{(\max )}=10^{-3}$.

TABLE I

PARAMETER SETting FOR WLAN CASE StUdy

\begin{tabular}{|c|c|}
\hline Parameter & Value \\
\hline \hline Primary Signal Bandwidth & 22MHz $(1 \mathrm{WLAN}$ Band $)$ \\
Primary Signal Power & $15 \mathrm{dBm}$ \\
Path Loss $(\eta)$ & 4.5 \\
Shadowing $\left(\mu, \sigma^{2}\right)$ & $0 \mathrm{dBm}, 10 \mathrm{~dB}$ \\
AWGN Power $\left(\sigma^{2}\right)$ & $-96 \mathrm{dBm}$ \\
Interference Limit $\left(P_{\mathcal{I}}^{(\max )}\right)$ & $10^{-3}$ \\
Total Sensing Time $\left(T_{s}\right)$ & $2.5 \mathrm{msec}$ \\
Sensed Band Size $\left(B_{W}\right)$ & $200 \mathrm{kHz}$ \\
Max. Number of Sensed Bands $\left(M_{\max }\right)$ & 100 \\
Signal Power in Sensed Band $\left(P_{W}\right)$ & $-5 \mathrm{dBm}$ \\
Prohibited Area Radius $\left(R_{\mathcal{I}}\right)$ & $300 \mathrm{~m}$ \\
\hline
\end{tabular}

\section{Performance evaluation}

Let us now evaluate the efficiency of limited sensing, that is, the achievable effective cognitive capacity, as a function of the network density. The capacity is derived through the numerical solution of the optimization problem in (1).

1) Parameter setting: As an example, we consider local primary and secondary networks, with transmission parameters comparable to IEEE 802.11x WLANs. Table I lists the input

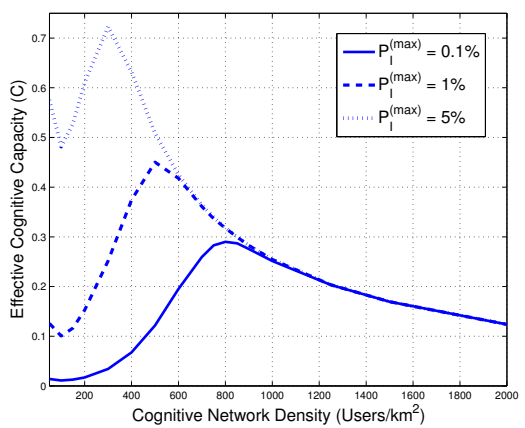

Fig. 4. Effective cognitive capacity with respect to average secondary network density and for various interference limits.

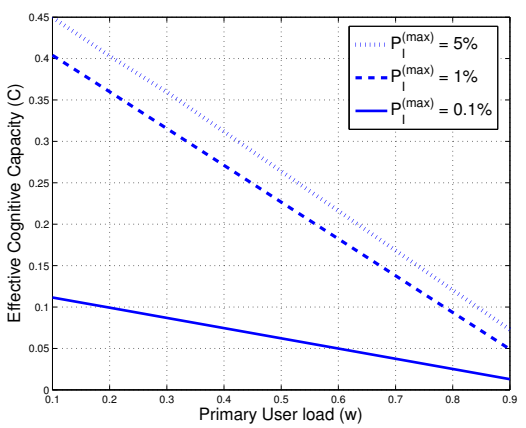

Fig. 6. Effective cognitive capacity as a function of primary user activity load, $w$, for various interference constraint values. User density: $\rho=500$ Users $/ \mathrm{Km}^{2}$

parameters for our numerical analysis, unless otherwise stated.

The particular value for $R_{\mathcal{I}}$, which defines the size of the prohibited area, is selected based on practical transmission ranges in wireless local area networks. $M_{\max }$ is chosen equal to 100 bands. Each of those narrow bands has a bandwidth of $B_{W}=200 \mathrm{kHz}$, as proposed in [28].

2) Numerical analysis: First we consider the case when the PU only occupies a single band and evaluate how $M-$ the number of bands sensed - affects the effective cognitive capacity. That is, $M$ is now input parameter of (1). Figure 3 shows, that $M$ is indeed a parameter to be optimized, since depending on the SU density $\rho$ there can be a local optimum $M<M_{\max }$. An expansion of $M$ behind this value increases the probability of false alarms, which leads to decreased per user cognitive capacity. After a local minimum, the capacity is expected to increase again, since in the unrealistic case of $M \rightarrow \infty$, the probability of interference tends to zero even without sensing, and consequently, the cognitive capacity tends to 1 .

The effective cognitive capacity, as a function of the secondary network density and for different interference limits is depicted in Figure 4. The capacity is determined via the numerical solution of (1). Under a given interference 
constraint, the cognitive network capacity reaches a highest value as network density increases, due to improved sensing efficiency. Above this "optimal" network density the capacity falls, as the now marginal improvement of sensing efficiency is not sufficient to accommodate the increasing need for bandwidth. For high user densities the cognitive capacity does not depend much on the interference limit, as a result of the high spectrum sensing performance. We observe a local minimum of cognitive capacity at low network densities. For densities below this value the sensing performance is weak, but as only few SUs are in the protected area, only few bands are accessed, and thus the probability of interference remains low.

Figure 5 shows the cognitive network capacity when the primary user operates in multiple bands. While the capacity decreases with the PU load, the the optimal network density, that is, where the capacity is maximized, does not seem to be affected. At the same time Figure 6 shows that the cognitive capacity decreases nearly linearly with the PU load, for the considered strict interference limits $P_{\mathcal{I}}^{(\max )}$.

Due to this independence, and for the sake of simplicity, we consider primary users operating in a single band in the rest of the paper. The extension for variable PU load is straightforward.

Our evaluation proves that the set of frequencies that can be sensed reliably by the SUs limits the achievable effective capacity in dense secondary networks. To overcome this limitation, the set of frequency bands available for cognitive transmission have to be extended, without the decrease of the per band sensing time of the SUs. This can be achieved by extended sensing, that is, by allowing the SUs to sense different subsets of the primary bands.

\section{Extended Spectrum Sensing}

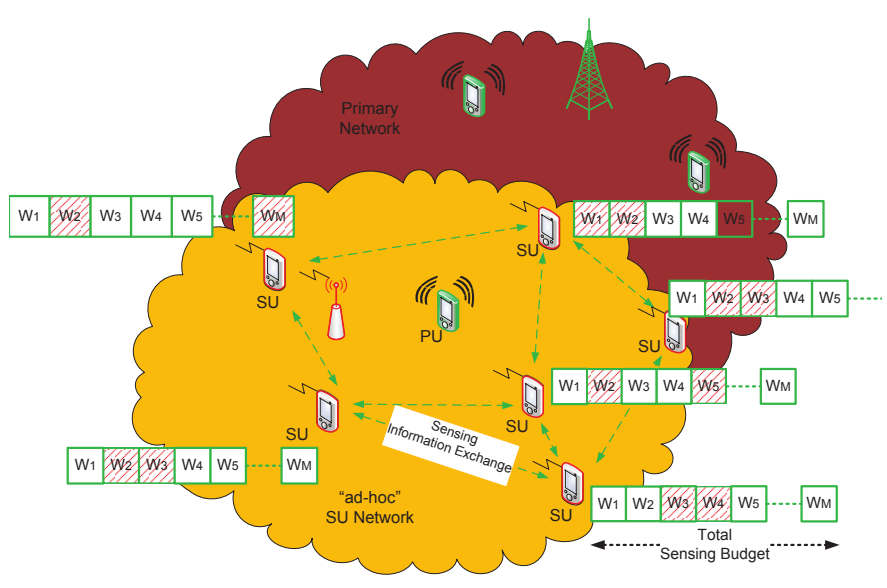

Fig. 7. The distribution of sensing duties in a dense cognitive network.

Let us now evaluate the performance of extended sensing. As defined in Section III-A, the SUs together sense a set of $\mathcal{W}$ primary bands, of size $|\mathcal{W}|=W$, giving the nominal sensing budget. This nominal sensing budget can be larger than the local sensing budget of the nodes, $\mathcal{M}_{i} \subset \mathcal{W}$ and $\left|\mathcal{M}_{i}\right|=$ $M \leq M_{\max }<W$. This situation is depicted in Figure 7. If all the users sense the same subset $\mathcal{M}$, the scenario is reduced to the one of Section IV and $\mathcal{M}_{i}=\mathcal{W}, \forall i$.

\section{A. Analytic model for extended spectrum sensing with different sensing policies}

1) Random sensing: In this case the secondary users randomly select the subset $\mathcal{M}_{i}$ of frequency bands to sense, independently from each other. An SU selects each of the spectrum bands with equal probability, that is, the probability that a band is sensed by an arbitrary $\mathrm{SU} i$ is:

$$
p_{W, i}=\frac{M}{W}=p_{W}, \forall i=1, \ldots, N_{\mathcal{A}} .
$$

Consider the band $V \in \mathcal{W}$ used by the primary system. With probability $p_{W}$ an arbitrary SU belongs to the $N_{\mathcal{A}}^{V}$ SUs that sense band $V$ and additional $M-1$ out of the remaining $W-1$ spectrum bands, while with probability $\left(1-p_{W}\right)$ it belongs to the rest $N_{\mathcal{A}}-N_{\mathcal{A}}^{V}$ SUs which sense $M$ out of the $W-1$ bands.

Let us introduce $p\left(j, k \mid N_{\mathcal{A}}\right)$, the probability that when $N_{\mathcal{A}}$ SUs perform sensing, $k$ of them sense band $V$ and $j$ bands in $\mathcal{W} \backslash V$ are available for transmission, that is, are correctly detected as free.

With $p\left(j, k \mid N_{\mathcal{A}}\right)$ we can express $P\left(\mathcal{I} \mid N_{\mathcal{A}}, N_{\mathcal{B}}\right)$, the probability of interference on band $V$, similarly to (13) - (15), as:

$$
\begin{aligned}
& P\left(\mathcal{I} \mid N_{\mathcal{A}}, N_{\mathcal{B}}\right)= \\
& \quad \sum_{j=0}^{W-1} \sum_{k=0}^{N_{\mathcal{A}}} \operatorname{Pr}\{\text { miss. det. } \mid k\} . \\
& \quad \cdot \operatorname{Pr}\left\{\text { use } \mid N_{\mathcal{A}}, N_{\mathcal{B}}, j\right\} p\left(j, k \mid N_{\mathcal{A}}\right)= \\
& =\sum_{j=0}^{W-1} \sum_{k=0}^{N_{\mathcal{A}}} p_{M D}(k) \min \left\{1, \frac{N_{\mathcal{A}}+N_{\mathcal{B}}}{j+1}\right\} p\left(j, k \mid N_{\mathcal{A}}\right) .
\end{aligned}
$$

Similarly, the variable $p\left(j, k \mid N_{\mathcal{A}}\right)$ helps us to express the cognitive capacity. We calculate $C\left(N_{\mathcal{A}}, N_{\mathcal{B}}\right)$ according to (19), with some changes. First, the missed detection probability $p_{M D}\left(N_{\mathcal{A}}\right)$ depends now on the number of SUs sensing channel $V, p_{M D}\left(N_{\mathcal{A}}\right)=\sum_{k=0}^{N_{\mathcal{A}}} p_{M D}(k) p(k)$, where $k$ is the number of SUs sensing a band, which follows a binomial distribution with parameters $N_{\mathcal{A}}$ and $p_{W}$. Similarly, we replace $P_{f r}(j)$ with $\sum_{k=0}^{N_{\mathcal{A}}} p\left(j, k \mid N_{\mathcal{A}}\right)$.

The direct calculation of $p\left(j, k \mid N_{\mathcal{A}}\right)$ suffers from combinatorial complexity due to the random band selection at the SUs. Therefore, we propose a recursive algorithm to calculate these probabilities, where we integrate the individual sensing results of each of the $N_{\mathcal{A}}$ SUs sequentially, exploiting that they are independent and stochastically identical.

We define the two-dimensional stochastic process, where state $\mathbb{S}_{j, k}^{(s)}$ defines the event that $j$ spectrum bands are available and $k$ users choose to sense band $V$, after the results of the first $s$ SUs are integrated. $\mathrm{S}_{j, k}^{(s)}$ denotes the probability of state $\mathbb{S}_{j, k}^{(s)}$, with $\sum_{n=0}^{W} S_{n, k}^{(s)}=1$. The vector $\boldsymbol{S}_{k}^{(s)} \in \mathbb{R}^{W}$ denotes the stochastic vector of the available spectrum bands, given $k$, after incorporating the sensing processes of the first $s$ SUs. 
In each iteration, the process can move the from a state $\mathbb{S}_{j, k}^{(s)}$ to states $\mathbb{S}_{j-n, k}^{(s+1)}$ and $\mathbb{S}_{j-n, k+1}^{(s+1)}$, based on whether the $s+1$-th user senses band $V$ and given the false alarm events that it may generate. The value of $n$, which indicates the additional number of bands that are "infected" by a false alarm event after incorporating the sensing from the $s+1$-th SU, is bounded by:

$$
\max \{0, l-(W-1-j)\} \leq n \leq \min \{l, j\},
$$

with $l$ denoting the number of the generated false alarm events by user $s+1$. Given that the false alarm probability is the same for any spectrum band, and considering that SUs select to sense any spectrum band with the same probability, we can express the conditional probability of $n$ new bands getting infected by false alarm as:

$$
\begin{aligned}
& \pi_{j} \rightarrow j-n \mid l= \\
& \quad=\left(\begin{array}{l}
l \\
n
\end{array}\right) \prod_{u=0}^{n-1} \frac{j-u}{W-1-u} \cdot \prod_{u=0}^{l-n-1}\left(1-\frac{j-n}{W-1-n-u}\right),
\end{aligned}
$$

which indicates that $n$ out of $l$ false alarms infect new bands, while the rest infect already infected bands. Clearly, for values of $n$ outside the bounds given in (29) the conditional transition probabilities are zero. The unconditioned transition probabilities are computed by averaging based on the probability mass function of the number of the false alarm events $l$, $p(l \mid L)=\left(\begin{array}{c}L \\ l\end{array}\right) \mathbf{p}_{f a}^{l}\left(1-\mathbf{p}_{f a}\right)^{L-l}$, where $L=M$ if SU $s+1$ does not sense band $V$ and $L=M-1$ otherwise:

$$
\begin{gathered}
\boldsymbol{\pi}_{j, k \rightarrow j-n, k}=\sum_{l=0}^{M} \pi_{j \rightarrow j-n \mid l} \cdot p(l \mid M) p_{W}, \\
\boldsymbol{\pi}_{j, k-1 \rightarrow j-n, k}=\sum_{l=0}^{M-1} \pi_{j \rightarrow j-n \mid l} \cdot p(l \mid M-1)\left(1-p_{W}\right) .
\end{gathered}
$$

The initial state vector of the recursion is: $\boldsymbol{S}_{0}^{(0)}=$ $(0,0, \ldots, 1)$. The state vector in step $s+1$ is calculated as:

$$
\begin{aligned}
& S_{j, k}^{(s+1)}=\sum_{i=0}^{W-1} \boldsymbol{\pi}_{i, k \rightarrow j, k} \cdot S_{i, k}^{(s)}+ \\
& \quad+\sum_{i=0}^{W-1} \boldsymbol{\pi}_{i, k-1 \rightarrow j, k} \cdot S_{i, k-1}^{(s)},
\end{aligned}
$$

or in matrix form:

$$
\boldsymbol{S}_{k}^{(s+1)}=\boldsymbol{S}_{k}^{(s)^{T}} \cdot \Pi+\boldsymbol{S}_{k-1}^{(s)}{ }^{T} \cdot \Pi^{V},
$$

where $\Pi, \Pi^{V} \in \mathbb{R}^{W \times W}$ are the transition matrices:

$$
\Pi=\left[\boldsymbol{\pi}_{i, k \rightarrow j, k}\right], \quad \Pi^{V}=\left[\boldsymbol{\pi}_{i, k-1 \rightarrow j, k}\right],
$$

$\forall i, j=0,1, \ldots, W-1$ and $\forall k=0,1, \ldots, N_{\mathcal{A}}$.

With the above recursive process we calculate $S_{j, k}^{\left(N_{\mathcal{A}}\right)}$, and set $p\left(j, k \mid N_{\mathcal{A}}\right)=S_{j, k}^{\left(N_{\mathcal{A}}\right)}$.

Considering the recursive method described above, the computation complexity of calculating $p\left(j, k \mid N_{\mathcal{A}}\right)$ is of the order of $O\left(N_{\mathcal{A}} \cdot M \cdot W\right)$. Moreover, due to the recursive nature of the method, we can calculate the state distribution for $N_{\mathcal{A}}=i+1 \mathrm{SUs}$ based on the already computed state distribution for $i$ SUs with a complexity of $O(M \cdot W)$. As a result, the iterative algorithm makes it possible to calculate the capacity and interference values with polynomial complexity.
2) Coordinated sensing: Under coordinated sensing the $N_{\mathcal{A}}$ SUs in the sensing area cooperate to select the individual local sensing budgets $\mathcal{M}_{i}, i=1, \ldots, N_{\mathcal{A}}$, such that each spectrum band is sensed by approximately the same number of secondary users, $N_{\mathcal{A} d} \approx N_{\mathcal{A}} /(W / M)=N_{\mathcal{A}} M / W$. The approximation is accurate for $N_{\mathcal{A}} \gg W / M$, which is expected in dense networks.

To calculate the probability of interference, $P(\mathcal{I})$, we can now directly apply (13) - (18), but considering cooperative sensing by $N_{\mathcal{A} d}$ SUs for each spectrum band. The missed detection probability becomes:

$$
p_{M D}\left(N_{\mathcal{A} d}\right)=\left(\mathbf{p}_{m d}\left(R_{c}, \gamma\right)\right)^{N_{\mathcal{A d}}},
$$

while the band utilization probability is:

$$
\operatorname{Pr}\left\{\text { use } \mid\left(N_{\mathcal{A}}, N_{\mathcal{B}}\right)\right\}=\sum_{j=0}^{W-1} \min \left\{1, \frac{N_{\mathcal{A}}+N_{\mathcal{B}}}{j+1}\right\} P_{f r}(j)
$$

with

$$
\begin{aligned}
& P_{f r}(j)= \\
& =\left(\begin{array}{c}
W-1 \\
j
\end{array}\right)\left(p_{F A}\left(N_{\mathcal{A} d}\right)\right)^{W-1-j}\left(1-p_{F A}\left(N_{\mathcal{A} d}\right)\right)^{j} .
\end{aligned}
$$

The effective cognitive capacity is again calculated according to (19), using $p_{M D}\left(N_{\mathcal{A d}}\right)$ and $P_{f r}(j)$ from the equations above.

3) Optimal sensing with dynamic sensing budget adjustment: Now the secondary network maintains information on the actual number of SUs in the area at each point in time, optimizes the system variables $W, M, R_{c}$ and $\gamma$ according to (2), and then allocates sensing duties as in the case of coordinated sensing. The effective cognitive capacity in this case is expected to outperform the ones of random and coordinated sensing at the cost of a higher control traffic overhead. $P\left(\mathcal{I} \mid \mathcal{N}_{\mathcal{A}}, \mathcal{N}_{\mathcal{B}}\right)$ and $C\left(N_{\mathcal{A}}, N_{\mathcal{B}}\right)$ can be calculated as for coordinated sensing.

\section{B. Capacity limits in highly dense cognitive networks}

Let us now investigate the asymptotic behavior of the effective cognitive capacity at very high SU densities. We detail the evaluation of the optimal sensing extension scheme, and summarize the results for the random and coordinated schemes.

In highly dense networks we can approximate the capacity, defined in (19), with respect to the total number of SUs in the prohibited area, $N=N_{\mathcal{A}}+N_{\mathcal{B}}$. Let us introduce $P_{f r}(j \mid N)$ and $P_{f r}\left(j \mid N_{\mathcal{A}}\right)$ as the probability of $j$ channels detected free given $N$ SUs in the prohibited area, and given $N_{\mathcal{A}}$ SUs in the sensing area, respectively. Given $N, N_{\mathcal{A}}$ has binomial distribution, with $\mathbf{q}_{N}\left(N_{\mathcal{A}}\right)=\left(\begin{array}{c}N \\ N_{\mathcal{A}}\end{array}\right)\left[\left(\frac{R_{c}}{R_{\mathcal{I}}}\right)^{2}\right]^{N_{\mathcal{A}}}\left[1-\left(\frac{R_{c}}{R_{\mathcal{I}}}\right)^{2}\right]^{N-N_{\mathcal{A}}}$.

Considering that for large $N p_{M D}\left(N_{\mathcal{A}}\right) \approx 0$ and approximating $\min \left\{1, \frac{j}{N}\right\}$ with $\frac{j}{N}$, we get:

$$
\begin{aligned}
\mathbf{C} & (N) \approx \sum_{j=0}^{W-1} \min \left\{1, \frac{j}{N}\right\} P_{f r}(j \mid N) \\
& \approx \sum_{j=0}^{W-1} j / N \cdot P_{f r}(j \mid N) \\
& =\sum_{N_{\mathcal{A}}=0}^{N} \sum_{j=0}^{W-1} j / N \cdot P_{f r}\left(j \mid N_{\mathcal{A}}\right) \mathbf{q}_{N}\left(N_{\mathcal{A}}\right) \\
& =\hat{\mathbf{C}}(N) .
\end{aligned}
$$


In the optimal sensing extension case $N_{\mathcal{A} d}=N_{\mathcal{A}} M / W$ SUs sense each of the bands, and thus $\hat{\mathbf{C}}(N)$ can be further approximated as:

$$
\begin{aligned}
& \hat{\mathbf{C}}_{o}(N)= \\
& \quad=\frac{W-1}{N} \sum_{N_{\mathcal{A}}=0}^{N}\left(1-\mathbf{p}_{f a}\right)^{N_{\mathcal{A d d}}} \mathbf{q}_{N}\left(N_{\mathcal{A}}\right) \\
& \quad \approx \frac{W-1}{N} \exp \left\{\left[\left(1-\mathbf{p}_{f a}\right)^{M / W}-1\right] N\left(\frac{R_{c}}{R_{\mathcal{I}}}\right)^{2}\right\} \\
& \approx \frac{W}{N} \exp \left\{\left[\left(1-\mathbf{p}_{f a}\right)^{M / W}-1\right] N\left(\frac{R_{c}}{R_{\mathcal{I}}}\right)^{2}\right\} .
\end{aligned}
$$

In dense networks, the probability that a band, that is detected free, is indeed allocated to an $\mathrm{SU}$ is close to one, that is, $\operatorname{Pr}\{$ use $\mid N\} \approx 1$. Based on this, we can approximate the probability of interference, defined in (13) - (15), as:

$$
\begin{aligned}
& P_{o}(\mathcal{I} \mid N) \approx \sum_{N_{\mathcal{A}}=0}^{N} \mathbf{p}_{m d}^{N_{\mathcal{A}} \cdot M / W} \mathbf{q}_{N}\left(N_{\mathcal{A}}\right) \approx \\
& \quad \approx \exp \left\{\left[\mathbf{p}_{m d}^{M / W}-1\right] N\left(\frac{R_{c}}{R_{\mathcal{I}}}\right)^{2}\right\}=\hat{P}_{o}(\mathcal{I} \mid N) .
\end{aligned}
$$

For $\hat{P}_{o}(\mathcal{I} \mid N)=P_{\mathcal{I}}^{(\max )}$ we can then express $W$ as:

$$
W=M \ln \left(\mathbf{p}_{m d}\right) / \ln \left(1+\frac{\ln \left(P_{\mathcal{I}}^{(\max )}\right)}{N\left(R_{c} / R_{\mathcal{I}}\right)^{2}}\right),
$$

which already shows that in highly dense networks the size of the nominal sensing budget $\mathcal{W}$ has to be increased with $N$, to achieve optimal performance.

Replacing (37) in (35) we get the cognitive capacity with respect to the local sensing parameters, $M, R_{c}$ and $\gamma$ (shown by (38) on the top of the page).

Proposition 1: In highly dense networks and with optimal sensing extension, the effective cognitive capacity asymptotically tends to the following limit:

$$
\lim _{N \rightarrow \infty} \hat{\mathbf{C}}_{o}(N)=M \frac{R_{c}^{2} / R_{\mathcal{I}}^{2} \ln \mathbf{p}_{m d}}{\ln P_{\mathcal{I}}^{(\max )}}\left(P_{\mathcal{I}}^{(\max )}\right)^{\frac{\ln \left(1-\mathbf{p}_{f a}\right)}{\ln \mathbf{p}_{m d}}} .
$$

Proof: The limit can be derived analytically from (38).

Proposition 2: The limit of the effective cognitive capacity given in (39) is an increasing function of $M$.

Proof: The gradient of (39), with respect to $M$ is always positive.

According to Proposition 2, the optimum $M$ is $M_{\max }$, and $W$ is given by (37). Therefore, the optimization problem to maximize the limit of the cognitive capacity under optimal sensing extension becomes two dimensional:

$$
\mathbf{C}_{o}=\max _{R_{c}, \gamma}\left\{M_{\max } \frac{R_{c}^{2} / R_{\mathcal{I}}^{2} \ln \mathbf{p}_{m d}}{\ln P_{\mathcal{I}}^{(\max )}} P_{\mathcal{I}}^{(\max ) \frac{\ln \left(1-\mathbf{p}_{f a}\right)}{\ln \mathbf{p}_{m d}}}\right\} .
$$

Furthermore, as this is now an unconstrained optimization problem, maximization can be performed sequentially for the variables of the system, thus reducing the complexity significantly:

$$
\begin{aligned}
& \mathbf{C}_{o}=M_{\max } /\left[R_{\mathcal{I}}^{2} \ln P_{\mathcal{I}}^{(\max )}\right] . \\
& \quad \cdot \max _{R_{c}}\left\{\max _{\gamma}\left\{R_{c}^{2} \ln \mathbf{p}_{m d} P_{\mathcal{I}}^{(\max ) \frac{\ln \left(1-\mathbf{p}_{f a}\right)}{\ln \mathbf{p}_{m d}}}\right\}\right\} .
\end{aligned}
$$

Corollary: The asymptotic cognitive capacity limit of optimal extended spectrum sensing depends on the input parameters such as the interference probability constraint and the size of the prohibited area, and on the performance of the local sensing, which in turn depends on the primary transmission power and the sensing time, but even on the optimal sensing area.

The process of deriving the approximate capacity and interference, and finally the capacity limits of random and coordinated sensing extension is similar. Specifically, for the coordinated scheme:

$$
\hat{\mathbf{C}}_{c}(N) \approx \frac{W-1}{\alpha_{c}(M) \pi \rho R_{\mathcal{I}}^{2}} e^{-\pi \rho R_{\mathcal{I}}^{2}}\left[e^{\alpha_{c}(M) \pi \rho R_{\mathcal{I}}^{2}}-1\right],
$$

where $\alpha_{c}(M)=e^{\left[\left(1-\mathbf{p}_{f a}\right)^{M /|\mathcal{W}|}-1\right]\left(\frac{R_{c}}{R_{\mathcal{I}}}\right)^{2}}$, and

$$
\hat{P}_{c}(\mathcal{I} \mid N)=\exp \left\{-\pi \rho R_{\mathcal{I}}^{2}\left(1-e^{\beta_{c}(M)}\right)\right\},
$$

where $\beta_{c}(M)=\left[\mathbf{p}_{m d}^{M / W}-1\right] \cdot\left(\frac{R_{c}}{R_{I}}\right)^{2}$.

For the random sensing scheme:

$$
\hat{\mathbf{C}}_{r}(N) \approx \frac{W-1}{\alpha_{r}(M) \pi \rho R_{\mathcal{I}}^{2}} e^{-\pi \rho R_{\mathcal{I}}^{2}}\left[e^{\alpha_{d}(M) \pi \rho R_{\mathcal{I}}^{2}}-1\right],
$$

where $\alpha_{r}(M)=\exp \left\{\left[e^{-\mathbf{p}_{f a} M / W}-1\right]\left(\frac{R_{c}}{R_{\mathcal{I}}}\right)^{2}\right\}$, and

$$
\hat{P}_{r}(\mathcal{I} \mid N)=\exp \left\{-\pi \rho R_{\mathcal{I}}^{2}\left(1-e^{\beta_{r}}\right)\right\},
$$

where $\beta_{r}=\left[\exp \left\{-\left(1-\mathbf{p}_{m d}\right) M / W\right\}-1\right]\left(R_{c} / R_{\mathcal{I}}\right)^{2}$.

\section{Performance evaluation}

In this section we evaluate the performance of extended sensing, using the parameters from Table I. The presented results are based on the numerical evaluation of (1) for random and cooperative sensing extension, (2) for optimal sensing extension and (41), (42) and (44) for the capacity limits of the different schemes.

We consider first the relationship between $\rho$, the average density of the cognitive network and the size of the nominal sensing budget $W$ that is required to maximize the effective cognitive capacity. This is an important design factor, as it determines the spectrum budget that should be "reserved" for the particular cognitive network to maximize spectrum efficiency.

Figure 8 depicts the optimal value of $W$ as a function of the density of the cognitive network, for the random and coordinated sensing extension schemes and the average of the optimal values for the optimal sensing scheme. For the optimal scheme $W$ increases linearly with the number of SUs. When we consider networks with high density the nominal budget required by the coordinated and random schemes has a similar linear behavior. For low or average densities, however, an increased frequency budget is required, to compensate for the reduced quality of cooperative sensing. The optimal sensing budget in the case of random sensing is lower compared to the required ones under coordinated and optimal sensing. This is explained by the need to keep the number of SUs sensing a band reasonably high, even for the bands that happen to be selected by relatively few SUs. 


$$
\hat{\mathbf{C}}_{o}(N)=\frac{M \ln \left(\mathbf{p}_{m d}\right)}{N \ln \left(1+\frac{\ln \left(P_{\mathcal{I}}^{(\max )}\right)}{N\left(\frac{R_{c}}{R_{\mathcal{I}}}\right)^{2}}\right)} \cdot \exp \left\{\left[\left(1-p_{f a}\right)^{\ln \left(1+\ln \left(P_{\mathcal{I}}^{(\max )}\right) / N\left(\frac{R_{c}}{R_{\mathcal{I}}}\right)^{2}\right) / \ln \left(\mathbf{p}_{m d}\right)}-1\right] N\left(\frac{R_{c}}{R_{\mathcal{I}}}\right)^{2}\right\}
$$

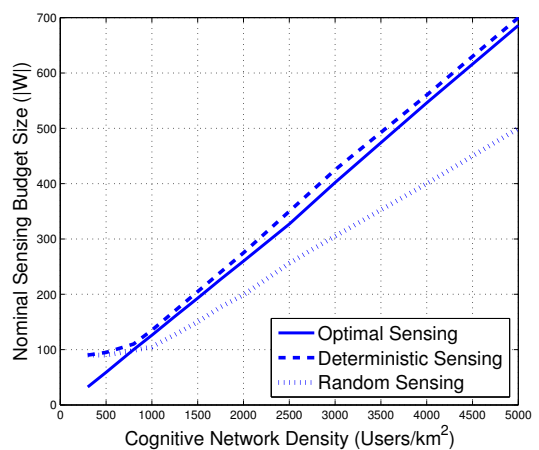

Fig. 8. Nominal Sensing Budget for highly dense cognitive networks, for the different sensing coordination schemes.

Figure 9 shows the effective cognitive capacity with respect to the average density of the cognitive network, together with the asymptotic limits at $\rho \rightarrow \infty$. We recall from Figure 4 that under the considered $P_{\mathcal{I}}^{(\max )}=10^{-3}$, limited sensing with $W=M$ achieved maximum performance of $\mathbf{C} \approx 0.28$ at a density of ca. 800 users $/ \mathrm{km}^{2}$. Considering sensing extension with random sensing we observe the same peak. The capacity then falls, since, as reflected in Figure 9, in this density region the nominal sensing budget can not be extended significantly. Finally, the capacity increases with the network density, approaching the limit very slowly. Under coordinated and optimal sensing, the achievable capacity increases monotonically, approaching the limit with decreasing rate.

Clearly, the optimal sensing scheme outperforms the other discussed policies with the random sensing being the worst of the three, as a result of lack of coordination of the individual sensing processes at the SUs. The gain of the optimal sensing over the coordinated one is however around 5\%, which may not justify the additional control and computational complexity.

Finally, we evaluate how the capacity limit is affected by the input parameters, that is, the interference probability constraint, $P_{\mathcal{I}}^{(\max )}$, and prohibited area radius, $R_{\mathcal{I}}$. We consider the optimal sensing extension scheme, but the behavior of the other schemes is similar. As shown in Figure 10, the capacity limit becomes larger than 1 under loose interference constraint and small prohibited area, which shows that our approximation in (34), $\min \left\{1, \frac{j}{N}\right\} \approx \frac{j}{N}$ is not tight in this region. The capacity limit is very sensitive to the size of the prohibited area; as seen from (41) it decreases with $1 / R_{\mathcal{I}}^{2}$, a result of the quadratic increase of interfering SUs that have to share the spectrum resources. The capacity limit with respect to $P_{\mathcal{I}}^{(\max )}$, however, increases slowly, especially for large prohibited areas. Increasing the probability constraint

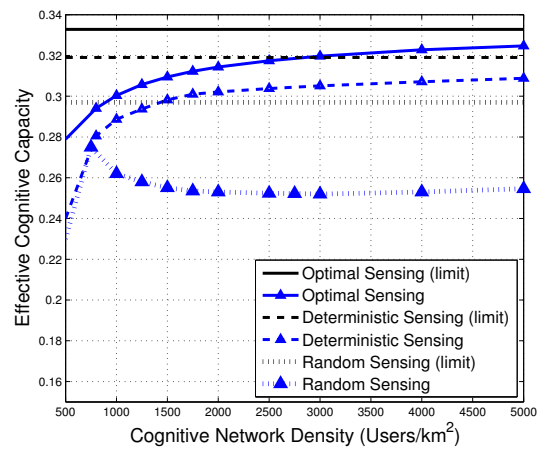

Fig. 9. Effective cognitive capacity for highly dense cognitive networks, for the different sensing coordination schemes.

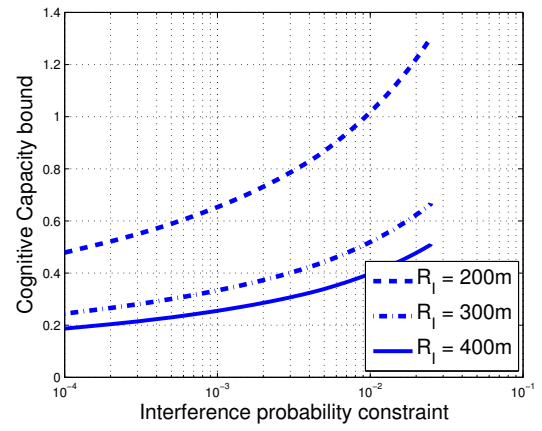

Fig. 10. Effective cognitive capacity limit with respect to the interference constraint imposed by the primary system and for different values of interference range.

with two orders of magnitude leads to less than doubled cognitive capacity limit.

\section{CONCLUSION}

In this paper we investigated the efficiency of spectrum sensing in dense cognitive networks, when the transmission characteristics of the primary and secondary systems are similar, and consequently the local spectrum sensing performance is highly distance dependent. We introduced the per user effective cognitive capacity as the performance metric, to reflect that the SUs performing the sensing also aim at utilizing the spectrum. We showed that the per user cognitive capacity decreases and approaches zero in dense networks if limited sensing is used, that is, all SUs sense the same set of bands.

We defined and evaluated different solutions to extend the set of sensed bands by letting each SU to sense only a subset of them. We have shown that extended sensing with coordination among the users leads to per user cognitive capacity that increases together with the SU density, converging to a limit 
which depends on the primary and secondary transmission characteristics, on the primary interference constraints and on the parameters of the cooperative sensing. Under random sensing allocation the capacity limit first falls as in the case of limited sensing, but then stabilizes and increases slightly with the SU density. Based on the numerical results we concluded that the performance gap of random and coordinated sensing is significant, while optimizing sensing based on the actual number of secondary nodes leads to little additional gain.

\section{REFERENCES}

[1] S. Geirhofer, Lang Tong, and B.M. Sadler. Dynamic spectrum access in the time domain: Modeling and exploiting white space. IEEE Communications Magazine, 45(5):66-72, 2007.

[2] M. Song, C. Xin, Y. Zhao, and X. Cheng. Dynamic spectrum access: from cognitive radio to network radio. IEEE Wireless Communications, 19(1):23-29, 2012.

[3] A. Sahai, N. Hoven, S. M. Mishra, and R. Tandra. Fundamental tradeoffs in robust spectrum sensing for opportunistic frequency reuse. Technical report, Berkeley, 2006.

[4] E. Visotsky, S. Kuffner, and R. Peterson. On Collaborative Detection of TV Transmissions in Support of Dynamic Spectrum Sharing. In IEEE Dynamic Spectrum Access Networks, 2005.

[5] A. Sahai, R. Tandra, and N. Hoven. Opportunistic spectrum use for sensor networks: the need for local cooperation. Berkeley Wireless Research Center, 2006.

[6] D. Duan, L. Yang, and J.C. Principe. Cooperative diversity of spectrum sensing for cognitive radio systems. IEEE Transactions on Signal Processing, 58(6):3218-3227, 2010.

[7] Ian F. Akyildiz, Won-Yeol Lee, and Kaushik R. Chowdhury. CRAHNs: Cognitive radio ad hoc networks. Ad Hoc Networks, 7(5):810 - 836, 2009.

[8] Z. Li, F.R. Yu, and M. Huang. A distributed consensus-based cooperative spectrum-sensing scheme in cognitive radios. IEEE Transactions on Vehicular Technology, 59(1):383-393, 2010.

[9] P. Di Lorenzo, S. Barbarossa, and A.H. Sayed. Decentralized resource assignment in cognitive networks based on swarming mechanisms over random graphs. IEEE Transactions on Signal Processing, 60(7):3755$3769,2012$.

[10] C. Cormio and K. R. Chowdhury. A survey on $\{\mathrm{MAC}\}$ protocols for cognitive radio networks. Ad Hoc Networks, 7(7):1315 - 1329, 2009.

[11] J. Xiang, Y. Zhang, and T. Skeie. Medium access control protocols in cognitive radio networks. Wireless Communications and Mobile Computing, 10(1):31-49, 2010.

[12] Y. Pei, A. T. Hoang, and Y.-C. Liang. Sensing-Throughput Tradeoff in Cognitive Radio Networks: How Frequently Should Spectrum Sensing be Carried Out? In IEEE International Symposium on Personal, Indoor and Mobile Radio Communications,(PIMRC'07), 2007.
[13] X. Xing, T. Jing, H. Li, Y. Huo, X. Cheng, and T. Znati. Optimal Spectrum Sensing Interval in Cognitive Radio Networks. IEEE Transactions on Parallel and Distributed Systems, 99(PrePrints), 2013.

[14] E.C.Y. Peh, Y.-C. Liang, Y. Liang Guan, and Y. Zeng. Optimization of cooperative sensing in cognitive radio networks: A sensing-throughput tradeoff view. IEEE Transactions on Vehicular Technology, 58(9):52945299, 2009

[15] R. Fan and H. Jiang. Optimal multi-channel cooperative sensing in cognitive radio networks. IEEE Transactions on Wireless Communications, 9(3), 2010.

[16] W. Han, J. Li, Z. Tian, and Y. Zhang. Efficient cooperative spectrum sensing with minimum overhead in cognitive radio. IEEE Transactions on Wireless Communications, 9(10):3006-3011, 2010.

[17] S. Chaudhari, J. Lunden, V. Koivunen, and H.V. Poor. Cooperative sensing with imperfect reporting channels: Hard decisions or soft decisions? IEEE Transactions on Signal Process., 60(1):18-28, 2012.

[18] C. Song and Q. Zhang. Cooperative spectrum sensing with multichannel coordination in cognitive radio networks. In IEEE International Conference on Communications, 2010.

[19] A.S. Cacciapuoti, I.F. Akyildiz, and L. Paura. Correlation-aware user selection for cooperative spectrum sensing in cognitive radio ad hoc networks. IEEE Journal on Selelected Topics of Signal Processing, 30(2):297-306, 2012

[20] B. F. Lo and I. F. Akyildiz. Reinforcement learning for cooperative sensing gain in cognitive radio ad hoc networks. Wireless Networks, 19(6):1237-1250, 2013.

[21] K. Koufos, K. Ruttik, and R. Jantti. Distributed sensing in multiband cognitive networks. IEEE Transactions on Wireless Communications, 10(5):1667-1677, 2011

[22] M.1 Timmers, S. Pollin, A. Dejonghe, A. Bahai, L. Van der Perre, and F. Catthoor. Accumulative interference modeling for distributed cognitive radio networks. Journal of Communications, 4(3), 2009.

[23] Jihoon Park, P. Pawelczak, P. Gronsund, and D. Cabric. Analysis framework for opportunistic spectrum ofdma and its application to the ieee 802.22 standard. IEEE Transactions on Vehicular Technology, 61(5):2271-2293, 2012.

[24] V. Fodor, I. Glaropoulos, and L. Pescosolido. Detecting low-power primary signals via distributed sensing to support opportunistic spectrum access. In IEEE International Conference on Communications, 2009.

[25] C. Taylor, A. Rahimi, J. Bachrach, H. Shrobe, and A. Grue. Simultaneous localization, calibration, and tracking in an ad hoc sensor network. In The Fifth International Conference on Information Processing in Sensor Networks, (IPSN'06), 2006.

[26] J. Gribben, A. Boukerche, and R. W. Nelem Pazzi. Scheduling for scalable energy-efficient localization in mobile ad hoc networks. In IEEE Communications Society Conference on Sensor Mesh and Ad Hoc Communications and Networks (SECON), 2010.

[27] I. Glaropoulos and V. Fodor. On the efficiency of spectrum sensing in adhoc cognitive radio networks. In ACM Mobicom Workshop on Cognitive Wireless Networking (CoRoNet), 2009.

[28] B. Mercier et al. Sensor networks for cognitive radio: Theory and system design. In ICT Mobile Summit, 2008. 